DEPT. OF MATH./CMA UNIV. OF OSLO

PURE MATHEMATICS NO 18

ISSN $0806-2439 \quad$ NOVEMBER 2010

\title{
A NOTE ON CONVERGENCE OF OPTION PRICES AND THEIR GREEKS FOR LÉVY MODELS
}

\author{
FRED ESPEN BENTH, GIULIA DI NUNNO, AND ASMA KHEDHER
}

\begin{abstract}
We study the robustness of option prices to model variation after a change of measure where the measure depends on the model choice. We consider geometric Lévy models in which the infinite activity of the small jumps is approximated by a scaled Brownian motion. For the Esscher transform, the minimal entropy martingale measure, the minimal martingale measure and the mean variance martingale measure, we show that the option prices and their corresponding deltas converge as the scaling of the Brownian motion part tends to zero. We give some examples illustrating our results.
\end{abstract}

\section{INTRODUCTION}

In incomplete markets, not every contingent claim can be replicated by a self-financing strategy. Instead of eliminating the risk by a perfect hedge, the issuer can adopt a partial hedging strategy according to some optimality criteria minimizing the risk exposure, and in the end bearing some of the risk (see e.g. Cont and Tankov [9] for more about pricing and hedging in incomplete markets).

In this paper, we consider an incomplete market where stock price fluctuations are modeled by a geometric Lévy process $S(t)=S(0) \exp (L(t))$, with $L$ being a Lévy process under the physical measure. Approximating the small jumps of the Lévy process $L$ with a Brownian motion scaled by the standard deviation of the small jumps, we can obtain another model for the dynamics of the stock price. This approximation was first introduced by Rydberg [19], and later analyzed rigourously by Asmussen and Rosinski [2]. From these papers, we know that the approximating stock price dynamics converges. The question is if the same holds true for the option prices and their Greeks under a risk-neutral equivalent martingale measure. In this paper, we show that this is indeed the case for the most popular choices of equivalent martingale measures. The problem we are facing here is that the choice of pricing measure is dependent on the approximation.

Due to market incompleteness for these models, there will exist infinitely many equivalent measures under which the discounted price processes are martingales. Gerber and Shiu [14, 15] proposed the Esscher transform as a potential pricing measure for Lévy models (see also Bühlmann et al. [7]). They explain their choice by modeling investor preferences by a power utility function and prove that in this case the investor's price when issuing an

Date: Revised February 28th, 2012.

Key words and phrases. Option pricing, equivalent martingale measure, Lévy process, delta hedging, robustness. 
option is given by the expected discounted payoff computed with respect to the Esscher measure.

Another popular choice is the minimal entropy martingale measure, which is the probability of having minimum relative entropy with respect to the market probability (see Goll and Rüschendorf [16]). Fujiwara and Miyahara [11] show that the minimal entropy martingale measure is given by an Esscher transformation for exponential Lévy models of the stock price dynamics.

The minimal martingale measure, first introduced by Föllmer and Sondermann [13] for martingales and later extended to the general semimartingale case by Föllmer and Schweizer [12], is defined via locally risk minimizing hedging strategies. One considers strategies which have a cost $C>0$. It turns out that the value process of a strategy that is minimizing locally the residual risk is given by the conditional expectation of the option's payoff under the minimal martingale measure. One drawback with this approach is the fact that one has to work with strategies which are not self-financing. If one prefers to avoid intermediate costs or unplanned income, a second idea is to insist on self-financing strategies that minimize the terminal hedging error in the mean-square sense. The meanvariance optimal measure is then used to calculate mean-variance optimal strategies (see Schweizer [21]).

Considering each of these equivalent measures, we prove that the option prices in the approximating model for the underlying stock converge to the prices derived on the stock dynamics modeled via the corresponding infinite activity Lévy process. This is an important consideration from the modeling point of view, as it is hard to decide which model for price dynamics is best between one where the small variations come from a jump process with infinite activity or from a continuous martingale (Brownian motion). By our results we have robustness in option prices and their Greeks with respect to this modeling choice. Moreover, in numerical procedures such an approximation comes in handy, since stability results are crucial for defending the approximation from an application point of view.

We study the stability of the delta of the option prices. Recall that the delta is defined as the sensitivity of the option price with respect to the state of the underlying asset. A convergence result for the delta of option prices was shown in Benth, Di Nunno, and Khedher [5] in the case of geometric Lévy processes, and later generalized to jump diffusions in Benth, Di Nunno, and Khedher [6], however, without accounting for the model dependency of the pricing measure. In this paper, we prove that after a change of measure the convergence result for the delta of option prices still holds true. In addition, we derive estimates for the rate of convergence in terms of the volatility of the small jumps in the infinite activity Lévy process.

The paper is organized as follows. In Sections 2, we make a short introduction about Lévy processes and present the geometric Lévy stock price model studied in this paper. In Section 3, we show the stability of option prices after a change of measure considering each of the following measures: the Esscher transform, the minimal entropy, the minimal martingale measure, and the mean-variance martingale measure. We illustrate our results with examples. 


\section{FRAMEWORK: TWO MODELS FOR THE STOCK PRICE DYNAMICS}

DEPT. OF MATH./CMA UNIV. OF OSLO

Let $(\Omega, \mathcal{F}, \mathbb{P})$ be a complete probability space equipped with a filtration $\left\{\mathcal{F}_{t}\right\}_{t \in[0, T]}(T>$ 0 ) satisfying the usual conditions (see Karatzas and Shreve [18]). We introduce the Lévy process $L=L(t), 0 \leq t \leq T$, on the given probability space and denote by $B=B(t)$, $0 \leq t \leq T$, a Brownian motion independent of $L$. We set $L(0)=B(0)=0$, and work with the right-continuous with left limits (also called càdlàg) version of the Lévy process, using the notation $\triangle L(t):=L(t)-L(t-)$. Denote the Lévy measure of $L$ by $\ell(d z)$. Recall that $\ell(d z)$ is a $\sigma$-finite Borel measure on $\mathbb{R}_{0}:=\mathbb{R}-\{0\}$.

The Lévy-Itô decomposition of a Lévy process will play an important role in our analysis, and we recall it here for the convenience of the reader (see Sato [20]):

Theorem 2.1. Let $L$ be a Lévy process on $\mathbb{R}$ and $\ell$ its Lévy measure. Then we have:

- $\ell$ verifies

$$
\int_{\mathbb{R}_{0}} \min \left(1, z^{2}\right) \ell(d z)<\infty
$$

- The jump measure of $L$, denoted by $N(d t, d z)$, is a Poisson random measure on $\left[0, \infty\left[\times \mathbb{R}_{0}\right.\right.$ with intensity measure $\ell(d z) d t$.

- There exists a Brownian motion $W(t), 0 \leq t \leq T$, and two constants $a, b \in \mathbb{R}$ such that

$$
L(t)=a t+b W(t)+Z(t)+\lim _{\varepsilon \downarrow 0} \widetilde{Z}_{\varepsilon}(t)
$$

where

$$
Z(t):=\sum_{s \in[0, t]} \triangle L(s) \mathbf{1}_{\{|\triangle L(s)| \geq 1\}}=\int_{0}^{t} \int_{|z| \geq 1} z N(d s, d z)
$$

and

$$
\widetilde{Z}_{\varepsilon}(t):=\sum_{s \in[0, t]} \Delta L(s) \mathbf{1}_{\{\varepsilon \leq|\triangle L(s)|<1\}}-t \int_{\varepsilon \leq|z|<1} z \ell(d z)=\int_{0}^{t} \int_{\varepsilon \leq|z|<1} z \tilde{N}(d s, d z),
$$

where $\tilde{N}(d t, d z):=N(d t, d z)-\ell(d z) d t$ is the compensated Poisson random measure of $L$. The convergence of $\widetilde{Z}_{\varepsilon}(t)$ in $(2.1)$ is almost sure and uniform on $t \in[0, T]$. The components $W, Z$ and $\widetilde{Z}_{\varepsilon}$ are independent.

In various applications involving statistical and numerical methods, it is often useful to approximate the small jumps by a scaled Brownian motion. This approximation was advocated in Rydberg [19] as a way to simulate the path of a Lévy process with normal inverse Gaussian (NIG) distributed increments and later studied in detail by Asmussen and Rosinski [2] for general Lévy models. We shall make use of it to study the robustness of option prices and their deltas based on exponential jump models. 
We introduce the following notation for the variation of the Lévy process $L$ close to the origin. For $0<\varepsilon \leq 1$, define

$$
\sigma^{2}(\varepsilon):=\int_{|z|<\varepsilon} z^{2} \ell(d z)
$$

Since every Lévy measure $\ell(d z)$ integrates $z^{2}$ in an open interval around zero, we have that $\sigma^{2}(\varepsilon)$ is finite for any $\varepsilon>0$. Note that the $\sigma^{2}(\varepsilon)$ is the variance of the jumps of $L$ smaller than $\varepsilon$ in the case $L$ is symmetric. By dominated convergence $\sigma^{2}(\varepsilon)$ converges to zero when $\varepsilon \downarrow 0$.

Inspired by the Lévy-Itô decomposition (2.1) we introduce now an approximating Lévy process (in law)

$$
L_{\varepsilon}(t):=a t+b W(t)+\sigma(\varepsilon) B(t)+Z(t)+\widetilde{Z}_{\varepsilon}(t),
$$

with $\sigma^{2}(\varepsilon)$ defined as in (2.2) and $B$ a Brownian motion independent of $L$ (which means independent of $W$ ). From the definition of $\widetilde{Z}_{\varepsilon}$, we see that we have substituted the small jumps (compensated by their expectation) in $L$ by a Brownian motion scaled with $\sigma(\varepsilon)$, the standard deviation of the compensated small jumps. We have the following result taken from Benth, Di Nunno, and Khedher [5].

Proposition 2.2. Let the processes $L$ and $L_{\varepsilon}$ be defined as in equation (2.1) and (2.3), respectively. Then, for every $t$,

$$
\lim _{\varepsilon \rightarrow 0} L_{\varepsilon}(t)=L(t) \quad \mathbb{P}-\text { a.s. }
$$

In fact, the limit above also holds in $L^{1}(\Omega, \mathcal{F}, \mathbb{P})$ with

$$
\mathbb{E}\left[\left|L_{\varepsilon}(t)-L(t)\right|\right] \leq 2 \sigma(\varepsilon) \sqrt{t} .
$$

We shall make use of this approximation and its convergence properties in our analysis. Let $S=S(t), 0 \leq t \leq T$, be a geometric Lévy process defined by

$$
S(t)=S(0) e^{L(t)}, \quad S(0)>0 .
$$

This represents a given stock price under the physical measure $\mathbb{P}$. We consider the discounted stock price process $\widehat{S}=\widehat{S}(t), 0 \leq t \leq T$, given by

$$
\widehat{S}(t)=e^{-r t} S(t), \quad \widehat{S}(0)=S(0)
$$

where the constant $r>0$ is the risk-free instantaneous interest rate. Assuming exponential integrability of the Lévy measure,

$$
\int_{1}^{\infty} \mathrm{e}^{z} \ell(d z)<\infty
$$

we apply the Itô formula, to represent the process $S$ as the solution of the following linear stochastic differential equation (SDE)

$$
S(t)=S(0)+\int_{0}^{t} S(s-) d \widehat{L}(s),
$$


where

$$
\widehat{L}(t)=a_{1} t+b W(t)+\int_{0}^{\text {DEPT. OF MATH./CMA UNIV. OF OSLO }} \int_{\mathbb{R}_{0}}^{\left(e^{z}-1\right) \tilde{N}(d s, d z) .}
$$

Here

$$
a_{1}=a+\frac{1}{2} b^{2}+\int_{\mathbb{R}_{0}}\left\{e^{z}-1-z \mathbf{1}_{|z| \leq 1}\right\} \ell(d z) .
$$

Using the Itô formula again, we can represent the discounted stock price $\widehat{S}$ as the solution of the following linear SDE

$$
d \widehat{S}(t)=\left(a_{1}-r\right) \widehat{S}(t-) d t+b \widehat{S}(t-) d W(t)+\widehat{S}(t-) \int_{\mathbb{R}_{0}}\left(e^{z}-1\right) \tilde{N}(d t, d z) .
$$

These representations will be useful in our later considerations.

As our second stock price dynamics $S_{\varepsilon}=S_{\varepsilon}(t), 0 \leq t \leq T$, is given by

$$
S_{\varepsilon}(t)=S(0) e^{L_{\varepsilon}(t)}, \quad S(0)>0,
$$

with $L_{\varepsilon}$ defined in (2.3). Thus, we have taken the dynamics $S(t)$ and substituted the small jumps of $L$ with a Brownian motion appropriately scaled. We note that by Prop. 2.2, $S_{\varepsilon}(t)$ converges $\mathbb{P}-$ a.s. to $S(t)$, for every $t$.

As we aim at studying the stability of option prices under a change of measure, we need to introduce the notion of (local) martingale measures for the discounted price process $\widehat{S}$. For this purpose, let $\mathcal{P}(\Omega, \mathcal{F})$ be the set of all probability measures on $(\Omega, \mathcal{F})$.

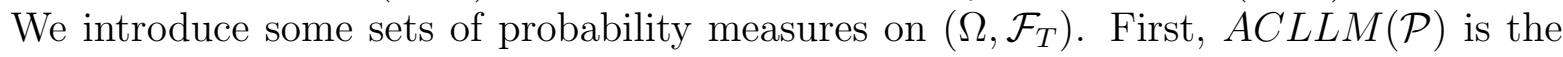
set of absolutely continuous local martingale measures,

$A C L M M(\mathcal{P}):=\left\{\widehat{\mathbb{P}} \in \mathcal{P}(\Omega, \mathcal{F}): \widehat{\mathbb{P}} \ll \mathbb{P}\right.$ on $\mathcal{F}_{T}$ and $\widehat{S}$ is a local martingale under $\left.\widehat{\mathbb{P}}\right\}$. Next, $\operatorname{EMM}(\mathcal{P})$ is the set of equivalent martingale measures for $\widehat{S}$,

$$
\operatorname{EMM}(\mathcal{P}):=\left\{\widehat{\mathbb{P}} \in \mathcal{P}(\Omega, \mathcal{F}): \widehat{\mathbb{P}} \sim \mathbb{P} \text { on } \mathcal{F}_{T} \text { and } \widehat{S} \text { is a martingale under } \widehat{\mathbb{P}}\right\} .
$$

We may introduce sets for $\widehat{S}_{\varepsilon}$ analogously.

The following theorem, due to Tankov [22], states the conditions for the absence of arbitrage in exponential Lévy models.

Theorem 2.3. Let $L$ be a Lévy process as defined in (2.1). The following statements are equivalent

(1) There exists a probability $\widetilde{\mathbb{P}}$ equivalent to $\mathbb{P}$ such that $L$ is a Lévy process under $\widetilde{\mathbb{P}}$ and $e^{L}$ is a martingale.

(2) Either $L=0$ or $L$ is not $\mathbb{P}$-a.s. monotone.

(3) One of the following conditions is satisfied:

- $b>0$.

- $b=0$ and $\int_{|x| \leq 1}|x| \ell(d x)=\infty$.

- $b=0, \int_{|x| \leq 1}|x| \ell(d x)<\infty, \ell((-\infty, 0))>0$ and $\ell((0, \infty))>0$.

- $b=0, \int_{|x| \leq 1}|x| \ell(d x)<\infty, \ell((-\infty, 0))>0$ and $a-\int_{|x| \leq 1} x \ell(d x)>0$. 


$$
\text { - } b=0, \int_{|x| \leq 1}|x| \ell(d x)<\infty, \ell((0, \infty))>0 \text { and } a-\int_{|x| \leq 1} x \ell(d x)<0 .
$$

In the following, we assume that our models do not allow for arbitrage.

\section{Stability of option PRices Under A CHANGe of MEASURE}

In this section we study the convergence of prices of options written on $S_{\varepsilon}$ to the corresponding prices written on $S$. We recall that our market models consists of a risk-free asset with instantaneous interest rate $r>0$ (used as numéraire) and a risky asset. We assume that $S$ represents the dynamics, under the physical mesure $\mathbb{P}$, of the risky asset on which it is written an option with payoff $f(S(t))$ at an exercise time $t$. Then the discounted expected value of $f(S(t))$ under some equivalent martingale measure is the option price. If alternatively we consider $S_{\varepsilon}$ as the price model of the risky asset, then the corresponding discounted risk-neutral expected value of $f\left(S_{\varepsilon}(t)\right)$ is the option price. Hence the price of the option depends on the choice of the model and, since the risk-neutral measures make the discounted price processes be martingales, then the option price depends also on the pricing measures (as they, in turn, depend on the chosen price dynamics model).

In the sequel we study whether the option prices are stable with respect to perturbation in the underlying dynamics when we substitute small jumps with an appropriate continuous martingale. Moreover we will consider the analysis of the stability of the Greeks. These are parameters of sensitivity of the option price to variations in the models descriptive elements. For example, the Delta and the Gamma evaluate, in different ways, the sensitivity

of the option price to the underlying initial price, the Vega is the sensitivity parameter to perturbation in volatility, etc. From a computational point of view, the Greeks are derivatives of the option price with respect to the parameter of interest.

In the analysis hereafter, we consider different choices of equivalent martingale measures widely used in the financial literature.

3.1. The Esscher transform. The moment generating function of $L(t)$, for any $t$, is given by

$$
\begin{aligned}
M_{t}(\theta) & =\mathbb{E}\left[e^{\theta L(t)}\right] \\
& =\exp \left\{t\left(a \theta+\frac{1}{2} b^{2} \theta^{2}+\int_{\mathbb{R}_{0}}\left(e^{\theta z}-1-z \mathbf{1}_{|z|<1} \theta\right) \ell(d z)\right)\right\}, \quad|\theta|<M,
\end{aligned}
$$

for some $0<M \leq \infty$ for which we have

$$
\int_{|z|>1} e^{\theta z} \ell(d z)<\infty, \quad|\theta|<M
$$

see Theorem 25.17 in Sato [20]. Set

$$
G(\theta):=a \theta+\frac{1}{2} b^{2} \theta^{2}+\int_{\mathbb{R}_{0}}\left(e^{\theta z}-1-z \mathbf{1}_{|z|<1} \theta\right) \ell(d z) .
$$


The Esscher transform is defined as a probability measure $\widetilde{\mathbb{P}}_{\theta} \sim \mathbb{P}$ (see Gerber and Shiu [14]) such that

$$
\begin{aligned}
\left.\frac{d \widetilde{\mathbb{P}}_{\theta}}{d \mathbb{P}}\right|_{\mathcal{F}_{t}} & =\exp (\theta L(t)-t G(\theta)) \\
& =\exp \left\{\theta b W(t)-\frac{1}{2} b^{2} \theta^{2} t+\theta \int_{0}^{t} \int_{\mathbb{R}_{0}} z \tilde{N}(d s, d z)-t \int_{\mathbb{R}_{0}}\left(e^{\theta z}-1-z \theta\right) \ell(d z)\right\} .
\end{aligned}
$$

We denote by $\widetilde{\mathbb{E}}_{\theta}$ the expectation under the new measure $\widetilde{\mathbb{P}}_{\theta}$.

In applications to finance, the risk neutral Esscher measure is defined as the $\widetilde{\mathbb{P}}_{\theta}$ such that the process $\widehat{S}(t)=e^{-r t} S(t), 0 \leq t \leq T$, is a martingale with respect to the filtration $\left\{\mathcal{F}_{t}\right\}_{t \in[0, T]}$. The condition

$$
\widetilde{\mathbb{E}}_{\theta}\left[e^{-r t} S(t)\right]=S(0)
$$

yields

$$
\mathbb{E}\left[e^{L(t)} e^{\theta L(t)-t G(\theta)}\right]=e^{r t}
$$

which is equivalent to

$$
G(\theta+1)-G(\theta)=r .
$$

Condition (3.3) is necessary and sufficient for $\widetilde{\mathbb{P}}_{\theta} \in E M M(\mathcal{P})$. From the definition of $G(\theta)$, we see that (3.3) becomes

$$
\begin{gathered}
a(1+\theta)+\frac{1}{2}(1+\theta)^{2} b^{2}+\int_{\mathbb{R}_{0}}\left\{e^{(\theta+1) z}-1-z \mathbf{1}_{|z|<1}(\theta+1)\right\} \ell(d z) \\
-a \theta-\frac{1}{2} \theta^{2} b^{2}-\int_{\mathbb{R}_{0}}\left\{e^{\theta z}-1-z \mathbf{1}_{|z|<1} \theta\right\} \ell(d z)=r
\end{gathered}
$$

Hence

$$
\begin{aligned}
a-r+b^{2} \theta+\frac{1}{2} b^{2} & +\int_{\mathbb{R}_{0}} e^{\theta z}\left(e^{z}-1-z \mathbf{1}_{|z|<1}\right) \ell(d z) \\
& +\int_{0<|z|<1} z\left(e^{\theta z}-1\right) \ell(d z)=0 .
\end{aligned}
$$

Define

$$
g(\theta):=b^{2} \theta+\int_{|z|>0} e^{\theta z}\left(e^{z}-1-z \mathbf{1}_{|z|<1}\right) \ell(d z)+\int_{0<|z|<1} z\left(e^{\theta z}-1\right) \ell(d z) .
$$

Under the arbitrage conditions, Gerber and Shiu [15] proved that equation (3.3) admits a unique solution in $\mathbb{R}$ if and only if one of these two conditions is fulfilled

- $M=\infty$,

- $M<\infty$ and $r-a-\frac{1}{2} b^{2} \in\left(\lim _{\theta \rightarrow-M} g(\theta), \lim _{\theta \rightarrow M} g(\theta)\right]$. 
We denote $\theta_{0}$ the solution of (3.3) and $\widetilde{\mathbb{P}}_{\theta_{0}}$ the corresponding Esscher measure. The stochastic process $L$ is still a Lévy process under the probability measure $\widetilde{\mathbb{P}}_{\theta_{0}}$. In this sense we say that the Esscher transform is structure preserving, see Theorem 33.1 in Sato [20]. The new characteristic triplet of $L$ under $\widetilde{\mathbb{P}}_{\theta_{0}}$ is given by $\left(b^{2}, \widetilde{\ell}, \widetilde{a}\right)$, where

$$
\tilde{\ell}(d z)=e^{\theta_{0} z} \ell(d z)
$$

and

$$
\widetilde{a}=a+b^{2} \theta_{0}+\int_{|z|<1} z\left(e^{\theta_{0} z}-1\right) \ell(d z) .
$$

Next, we consider the approximated price process $S_{\varepsilon}(t)$ and its discounted version $\widehat{S}_{\varepsilon}(t)=$ $e^{-r t} S_{\varepsilon}(t), 0 \leq t \leq T$. We define

$$
G_{\varepsilon}(\theta):=a \theta+\frac{1}{2} \theta^{2}\left(b^{2}+\sigma^{2}(\varepsilon)\right)+\int_{|z| \geq \varepsilon}\left(e^{\theta z}-1-z \mathbf{1}_{|z|<1} \theta\right) \ell(d z) .
$$

Note that for $G_{\varepsilon}(\theta)$ to exist, the Condition (3.2) is still sufficient. An Esscher probability measure $\widetilde{\mathbb{P}}_{\theta}^{\varepsilon} \sim \mathbb{P}$ is given by

$$
\begin{aligned}
\left.\frac{d \widetilde{\mathbb{P}}_{\theta}^{\varepsilon}}{d \mathbb{P}}\right|_{\mathcal{F}_{t}}= & \exp \left(\theta L_{\varepsilon}(t)-t G_{\varepsilon}(\theta)\right) \\
= & \exp \left\{\theta(b W(t)+\sigma(\varepsilon) B(t))-\frac{1}{2}\left(b^{2}+\sigma^{2}(\varepsilon)\right) \theta^{2} t+\theta \int_{0}^{t} \int_{|z| \geq \varepsilon} z \tilde{N}(d s, d z)\right. \\
& \left.\quad-t \int_{|z| \geq \varepsilon}\left(e^{\theta z}-1-z \theta\right) \ell(d z)\right\} .
\end{aligned}
$$

By the same argument as above, we can see that $\widetilde{\mathbb{P}}_{\theta}^{\varepsilon}$ is a risk-neutral equivalent martingale measure if and only if the parameter $\theta$ satisfies

$$
G_{\varepsilon}(\theta+1)-G_{\varepsilon}(\theta)=r .
$$

As in Gerber and Shiu [15], one can prove the existence and uniqueness of the parameter $\theta_{\varepsilon}$ solving $(3.6)$, for $\varepsilon$ fixed in $(0,1)$. We adapt their proof to our model.

Lemma 3.1. Define

$$
g_{\varepsilon}(\theta):=\left(b^{2}+\sigma^{2}(\varepsilon)\right) \theta+\int_{|z| \geq \varepsilon} e^{\theta z}\left(e^{z}-1-z \mathbf{1}_{|z|<1}\right) \ell(d z)+\int_{\varepsilon \leq|z|<1} z\left(e^{\theta z}-1\right) \ell(d z) .
$$

Then, for each $\varepsilon \in(0,1)$ the solution of

$$
G_{\varepsilon}(1+\theta)-G_{\varepsilon}(\theta)=r
$$

exists and is unique in $\mathbb{R}$ if and only if one of the following two conditions is satisfied

$$
\begin{gathered}
M=\infty \\
M<\infty \quad \text { and } r-a-\frac{1}{2}\left(\sigma^{2}(\varepsilon)+b^{2}\right) \in\left(\lim _{\theta \rightarrow-M} g_{\varepsilon}(\theta), \lim _{\theta \rightarrow M} g_{\varepsilon}(\theta)\right] .
\end{gathered}
$$


We denote this solution $\theta_{\varepsilon}$ emphasizing the dependence on $\varepsilon \in(0,1)$.

DEPT. OF MATH./CMA UNIV. OF OSLO

Proof. By dominated convergence for $\varepsilon$ fixed, the function $g_{\varepsilon}(\theta)$ is differentiable with derivative given by

$$
g_{\varepsilon}^{\prime}(\theta)=\left(b^{2}+\sigma^{2}(\varepsilon)\right)+\int_{|z| \geq \varepsilon} z\left(e^{z}-1\right) e^{\theta z} \ell(d z) .
$$

Note that $z(\exp (z)-1)>0$ when $|z| \geq \varepsilon$. Hence, since $g_{\varepsilon}^{\prime}(\theta) \geq \sigma^{2}(\varepsilon)>0$, it follows that $g_{\varepsilon}(\theta)$ is a strictly increasing function. Moreover, $g_{\varepsilon}(+\infty)=+\infty$ and $g_{\varepsilon}(-\infty)=-\infty$. Therefore, the equation $g_{\varepsilon}(\theta)+a-r+\frac{1}{2}\left(b^{2}+\sigma^{2}(\varepsilon)\right)=0$ admits a unique solution if and only if one of the conditions (3.7) or (3.8) is satisfied.

The stochastic process $L_{\varepsilon}$ is still a Lévy process under the probability measure $\widetilde{\mathbb{P}}_{\theta_{\varepsilon}}^{\varepsilon}$, with characteristic triplet given by $\left(b^{2}+\sigma^{2}(\varepsilon), \widetilde{\ell}^{\varepsilon}, \widetilde{a}^{\varepsilon}\right)$, for

$$
\tilde{\ell}_{\varepsilon}(d z)=e^{\theta_{\varepsilon} z} \ell(d z)
$$

and

$$
\widetilde{a}_{\varepsilon}=a+\left(b^{2}+\sigma^{2}(\varepsilon)\right) \theta_{\varepsilon}+\int_{\varepsilon \leq|z|<1} z\left(e^{\theta_{\varepsilon} z}-1\right) \ell(d z) .
$$

In the sequel, we need the following technical lemma in which we study the behavior of $\theta_{\varepsilon}$ when $\varepsilon$ goes to 0 . Recall that $\theta_{0} \in \mathbb{R}$ is the solution of (3.6).

Lemma 3.2. The parameter $\theta_{\varepsilon}$ is bounded uniformly in $\varepsilon, \varepsilon \in(0,1)$, and

$$
\left|\theta_{\varepsilon}-\theta_{0}\right| \leq C_{\theta_{0}} \sigma^{2}(\varepsilon)
$$

for a positive constant $C_{\theta_{0}}$ depending on $\theta_{0}$.

Proof. Recall the definition of $g_{\varepsilon}(\theta)$ in Lemma 3.1. In the proof of Lemma 3.1 we showed that $g_{\varepsilon}(\theta)$ is differentiable. Moreover, it is increasing in $\theta$. Therefore, the inverse $g_{\varepsilon}^{-1}(\theta)$ exists, it is differentiable and its derivative is given by $\left(g_{\varepsilon}^{-1}\right)^{\prime}(\theta)=\frac{1}{g_{\varepsilon}^{\prime}(\theta)}$. In the case when $b>0$, we have

$$
\begin{aligned}
g_{\varepsilon}^{\prime}(\theta) & =b^{2}+\sigma^{2}(\varepsilon)+\int_{|z| \geq \varepsilon} z e^{\theta z}\left(e^{z}-1\right) \ell(d z) \\
& \geq b^{2}
\end{aligned}
$$

Hence $\left(g_{\varepsilon}^{-1}\right)^{\prime}(\theta) \leq \frac{1}{b^{2}}$. By equations (3.3) and (3.6), we know that $\theta_{\varepsilon}$ and $\theta_{0}$ satisfy the following equations

$$
g_{\varepsilon}\left(\theta_{\epsilon}\right)=r-a-\frac{1}{2}\left(b^{2}+\sigma^{2}(\varepsilon)\right)
$$

and

$$
g_{\varepsilon}\left(\theta_{0}\right)=r-a-\frac{1}{2} b^{2}+\sigma^{2}(\varepsilon) \theta_{0}-\int_{|z|<\varepsilon} e^{\theta_{0} z}\left(e^{z}-1-z \mathbf{1}_{|z|<1}\right) \ell(d z)-\int_{|z|<\varepsilon} z\left(e^{\theta_{0} z}-1\right) \ell(d z),
$$


respectively. It follows that

$$
\begin{aligned}
\left|\theta_{\varepsilon}-\theta_{0}\right|=\mid & g_{\varepsilon}^{-1}\left(r-a-\frac{1}{2}\left(b^{2}+\sigma^{2}(\varepsilon)\right)\right)-g_{\varepsilon}^{-1}\left(r-a-\frac{1}{2} b^{2}+\sigma^{2}(\varepsilon) \theta_{0}\right. \\
& \left.-\int_{|z|<\varepsilon} e^{\theta_{0} z}\left(e^{z}-1-z \mathbf{1}_{|z|<1}\right) \ell(d z) \mid-\int_{|z|<\varepsilon} z\left(e^{\theta_{0} z}-1\right) \ell(d z)\right) \mid .
\end{aligned}
$$

The mean value theorem leads to

$$
\begin{aligned}
\left|\theta_{\varepsilon}-\theta_{0}\right| & \leq \frac{1}{b^{2}}\left|-\frac{1}{2} \sigma^{2}(\varepsilon)-\sigma^{2}(\varepsilon) \theta_{0}+\int_{|z|<\varepsilon}\left\{e^{\theta_{0} z}\left(e^{z}-1\right)-z\right\} \ell(d z)\right| \\
& =\frac{1}{b^{2}}\left|-\frac{1}{2} \sigma^{2}(\varepsilon)-\sigma^{2}(\varepsilon) \theta_{0}+\int_{|z|<\varepsilon}\left\{e^{z\left(\theta_{0}+1\right)}-1-z\left(\theta_{0}+1\right)\right\} \ell(d z)\right| \\
& -\int_{|z|<\varepsilon}\left\{e^{z \theta_{0}}-1-z \theta_{0}\right\} \ell(d z) \mid .
\end{aligned}
$$

We define $h(\theta)=\int_{|z|<\varepsilon}\left\{e^{z \theta}-1-z \theta\right\} \ell(d z)$. The function $h$ is differentiable and its derivative is given by $h^{\prime}(\theta)=\int_{|z|<\varepsilon} z\left(e^{\theta z}-1\right) \ell(d z)$. Thus, applying the mean value theorem to the function $h(\theta)$ and then to the function $f(\theta)=e^{\theta z}$, we get

$$
\begin{aligned}
\left|\theta_{\varepsilon}-\theta_{0}\right| & \leq \frac{1}{b^{2}}\left(\frac{1}{2} \sigma^{2}(\varepsilon)+\sigma^{2}(\varepsilon)\left|\theta_{0}\right|+\int_{|z|<\varepsilon}|z|\left|e^{\theta_{0} z}-1\right| \ell(d z) \mid\right. \\
& \leq \frac{1}{b^{2}}\left|\frac{1}{2} \sigma^{2}(\varepsilon)+\sigma^{2}(\varepsilon)\right| \theta_{0}\left|+\int_{|z|<\varepsilon}\right| z^{2}\left|e^{\theta_{0} z} \ell(d z)\right| \\
& \leq \frac{1}{b^{2}}\left|\frac{1}{2} \sigma^{2}(\varepsilon)+\sigma^{2}(\varepsilon)\right| \theta_{0}\left|+e^{\left|\theta_{0}\right|} \sigma^{2}(\varepsilon)\right| \\
& =C_{\theta_{0}} \sigma^{2}(\varepsilon) .
\end{aligned}
$$

Moreover,

$$
\left|\theta_{\varepsilon}\right| \leq M \wedge\left(\left|\theta_{0}\right|+C_{\theta_{0}} \sigma^{2}(\varepsilon)\right) \leq\left|\theta_{0}\right|+C_{\theta_{0}} \sigma^{2}(1)
$$

In the case when $b=0$ and $\theta>\theta_{0}$, we have $g_{\varepsilon}^{\prime}(\theta) \geq \int_{z \geq 1} z e^{\theta_{0} z}\left(e^{z}-1\right) \ell(d z)$ and therefore

$$
\left|\theta_{\varepsilon}-\theta_{0}\right| \leq \frac{1}{\int_{z \geq 1} z e^{\theta_{0} z}\left(e^{z}-1\right) \ell(d z)}\left|\frac{1}{2} \sigma^{2}(\varepsilon)+\sigma^{2}(\varepsilon)\right| \theta_{0}\left|+e^{\left|\theta_{0}\right|} \sigma^{2}(\varepsilon)\right| .
$$

When $b=0$ and $\theta<\theta_{0}$, we have $g_{\varepsilon}^{\prime}(\theta) \geq \int_{z \leq-1} z e^{\theta_{0} z}\left(e^{z}-1\right) \ell(d z)$ and in this case,

$$
\left|\theta_{\varepsilon}-\theta_{0}\right| \leq \frac{1}{\int_{z \leq-1} z e^{\theta_{0} z}\left(e^{z}-1\right) \ell(d z)}\left|\frac{1}{2} \sigma^{2}(\varepsilon)+\sigma^{2}(\varepsilon)\right| \theta_{0}\left|+e^{\left|\theta_{0}\right|} \sigma^{2}(\varepsilon)\right| .
$$

Therefore the result also holds in the case when $b=0$. 
Lemma 3.3. Let $\phi_{L_{\varepsilon}(T)}$ and $\phi_{L(T)}$ be the characteristic functions of $L_{\varepsilon}(T)$ under $\widetilde{\mathbb{P}}_{\theta_{\varepsilon}}^{\varepsilon}$, and $L(T)$ under $\widetilde{\mathbb{P}}_{\theta}$, respectively. Then we have DEPT. OF MATH./CMA UNIV. OF OSLO

$$
\lim _{\varepsilon \longrightarrow 0} \phi_{L_{\varepsilon}(T)}(u)=\phi_{L(T)}(u)
$$

for every $u \in \mathbb{R}$.

Proof. The characteristic function of $L_{\varepsilon}(T)$ under $\widetilde{\mathbb{P}}_{\theta_{\varepsilon}}^{\varepsilon}$ is given by

$$
\phi_{L_{\varepsilon}(T)}(u)=\exp \left\{\mathrm{i} \widetilde{a}_{\varepsilon} u-\frac{1}{2}\left(b^{2}+\sigma^{2}(\varepsilon)\right) u+\int_{|z| \geq \varepsilon}\left(e^{\mathrm{i} u z}-1-\mathrm{i} u z 1_{|z|<1}\right) \tilde{\ell}_{\varepsilon}(d z)\right\} .
$$

As $\theta_{\varepsilon}$ is bounded uniformly in $\varepsilon$, by Prop. 2.24 in Folland [10], we can take the limit inside the integral in equation (3.11) and then the result follows.

Let us now consider $f \in L^{1}(\mathbb{R})$, that is, the space of integrable functions on the real line. The Fourier transform of $f$ is defined by

$$
\widehat{f}(u)=\int_{\mathbb{R}} f(y) e^{\mathrm{i} u y} d y .
$$

Suppose in addition that $\widehat{f} \in L^{1}(\mathbb{R})$. Then the inverse Fourier transform is well-defined, and we have

$$
f(y)=\frac{1}{2 \pi} \int_{\mathbb{R}} e^{-\mathrm{i} u y} \widehat{f}(u) d u .
$$

With these two definitions at hand, we can do the following calculation taken from Carr and Madan [8]. Assume for every $x \in \mathbb{R}$ that $f(x+\cdot)$ is integrable with respect to the distribution $\widetilde{p}_{L_{\varepsilon}(T)}(d y)$ of $L_{\varepsilon}(T)$ under the measure $\widetilde{\mathbb{P}}_{\theta_{\varepsilon}}^{\varepsilon}$. Then

$$
\widetilde{\mathbb{E}}_{\theta_{\varepsilon}}\left[f\left(x+L_{\varepsilon}(T)\right)\right]=\int_{\mathbb{R}} f(x+y) \widetilde{p}_{L_{\varepsilon}(T)}(d y) .
$$

Invoking the representation of $f$ in (3.13), and applying Fubini-Tonelli to commute the integrations, we find

$$
\begin{aligned}
\widetilde{\mathbb{E}}_{\theta_{\varepsilon}}\left[f\left(x+L_{\varepsilon}(T)\right)\right] & =\int_{\mathbb{R}}\left\{\frac{1}{2 \pi} \int_{\mathbb{R}} e^{-i(x+y) u} \widehat{f}(u) d u\right\} \widetilde{p}_{L_{\varepsilon}(T)}(d y) \\
& =\frac{1}{2 \pi} \int_{\mathbb{R}} e^{-i u x}\left\{\int_{\mathbb{R}} e^{-i u y} \widetilde{p}_{L_{\varepsilon}(T)}(d y)\right\} \widehat{f}(u) d u .
\end{aligned}
$$

Thus, it follows that

$$
\widetilde{\mathbb{E}}_{\theta_{\varepsilon}}\left[f\left(x+L_{\varepsilon}(T)\right)\right]=\frac{1}{2 \pi} \int_{\mathbb{R}} e^{-i u x} \phi_{L_{\varepsilon}(T)}(-u) \widehat{f}(u) d u,
$$

where $\phi_{L_{\varepsilon}(T)}$ is the characteristic function of $L_{\varepsilon}(T)$ defined by equation (3.11).

In the setting presented so far, we can conclude the following result which gives the stability of option prices under the Esscher transform. 
Proposition 3.4. It holds that

$$
\lim _{\varepsilon \rightarrow 0} \widetilde{\mathbb{E}}_{\theta_{\varepsilon}}\left[f\left(x+L_{\varepsilon}(T)\right]=\widetilde{\mathbb{E}}_{\theta_{0}}[f(x+L(T)] .\right.
$$

In particular, if $\int_{\mathbb{R}}|\widehat{f}(u)|\left(|u|+|u|^{2}\right) d u<\infty$, then we have that the rate of convergence is

$$
\mid \widetilde{\mathbb{E}}_{\theta_{\varepsilon}}\left[f\left(x+L_{\varepsilon}(T)\right]-\widetilde{\mathbb{E}}_{\theta_{0}}[f(x+L(T))] \mid \leq \sigma^{2}(\varepsilon) C_{\theta_{0}}\right.
$$

where $C_{\theta_{0}}$ is a positive constant depending on $\theta_{0}$.

Proof. From the Fourier representation of the option prices, we estimate

$$
\begin{aligned}
\mid \widetilde{\mathbb{E}}_{\theta_{\varepsilon}}\left[f\left(x+L_{\varepsilon}(T)\right]\right. & -\widetilde{\mathbb{E}}_{\theta_{0}}[f(x+L(T))] \mid \\
& =\left|\frac{1}{2 \pi} \int_{\mathbb{R}}\left\{e^{-i u x} \phi_{L_{\varepsilon}(T)}(-u) \widehat{f}(u)-e^{-i u x} \phi_{L(T)}(-u) \widehat{f}(u)\right\} d u\right| \\
& \leq \frac{1}{2 \pi} \int_{\mathbb{R}}|\widehat{f}(u)|\left|\phi_{L_{\varepsilon}(T)}(-u)-\phi_{L(T)}(-u)\right| d u .
\end{aligned}
$$

Applying the mean value theorem to the function $u(x)=e^{i x}$, we get

$$
\begin{aligned}
& \left|\phi_{L_{\varepsilon}(T)}(-u)-\phi_{L(T)}(-u)\right| \\
& \leq \mid i \widetilde{a}_{\varepsilon} u-\frac{1}{2}\left(b^{2}+\sigma^{2}(\varepsilon)\right) u+\int_{|z| \geq \varepsilon}\left\{e^{i u z}-1-i u z \mathbf{1}_{|z|<1}\right\} \widetilde{\ell}_{\varepsilon}(d z) \\
& \quad-i \widetilde{a} u+\frac{1}{2} b^{2} u+\int_{\mathbb{R}_{0}}\left\{e^{i u z}-1-i u z \mathbf{1}_{|z|<1}\right\} \widetilde{\ell}(d z) \mid \\
& \leq|u|\left|\widetilde{a}_{\varepsilon}-\widetilde{a}\right|+\frac{1}{2} \sigma^{2}(\varepsilon)|u|+\left|\int_{|z|>\varepsilon}\left\{e^{i u z}-1-i u z \mathbf{1}_{|z|<1}\right\}\left\{e^{\theta_{\varepsilon} z}-e^{\theta_{0} z}\right\} \ell(d z)\right| \\
& \quad+\left|\int_{|z| \leq \varepsilon}\left\{e^{i u z}-1-i u z \mathbf{1}_{|z|<1}\right\} e^{\theta_{0} z} \ell(d z)\right|
\end{aligned}
$$

From the expressions of $\widetilde{a}_{\varepsilon}$ and $\widetilde{a}$, in (3.4) and (3.9), respectively, we have

$$
\begin{aligned}
\mid \phi_{L_{\varepsilon}(T)}(-u)- & \phi_{L(T)}(-u) \mid \\
\leq \mid & u \mid\left(b^{2}\left|\theta_{\varepsilon}-\theta_{0}\right|+\sigma^{2}(\varepsilon)\left|\theta_{\varepsilon}\right|+\left|\int_{\varepsilon \leq|z|<1} z\left(e^{\theta_{\varepsilon}}-e^{\theta_{0} z}\right) \ell(d z)\right|\right. \\
& \left.+\left|\int_{|z| \leq \varepsilon} z\left(e^{\theta_{0} z}-1\right) \ell(d z)\right|\right) \\
& +\frac{1}{2} \sigma^{2}(\varepsilon)|u|+\left|\int_{|z| \geq \varepsilon}\left\{e^{i u z}-1-i u z \mathbf{1}_{|z|<1}\right\}\left\{e^{\theta_{\varepsilon} z}-e^{\theta_{0} z}\right\} \ell(d z)\right| \\
& +\left|\int_{|z| \leq \varepsilon}\left\{e^{i u z}-1-i u z \mathbf{1}_{|z|<1}\right\} e^{\theta_{0} z} \ell(d z)\right|
\end{aligned}
$$

The mean value theorem leads to

$$
\left|\phi_{L_{\varepsilon}(T)}(-u)-\phi_{L(T)}(-u)\right|
$$




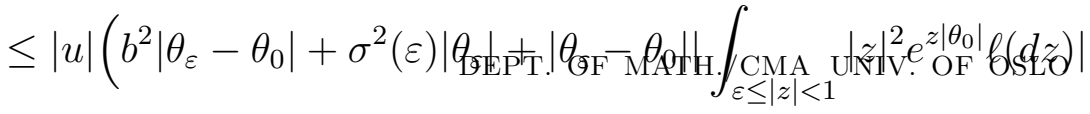

$$
\begin{aligned}
& \left.+\left|\int_{|z| \leq \varepsilon} z\left(e^{\theta_{0} z}-1\right) \ell(d z)\right|\right)+\frac{1}{2} \sigma^{2}(\varepsilon)|u| \\
& +\left|\theta_{\varepsilon}-\theta_{0}\right| \int_{|z| \geq \varepsilon}\left|e^{i u z}-1-i u z \mathbf{1}_{|z|<1}\right||z| e^{\left|\theta_{0}\right| z} \ell(d z) \\
& +\left|\int_{|z| \leq \varepsilon}\left(e^{i u z}-1-i u z\right) \ell(d z)\right| .
\end{aligned}
$$

From Lemma 3.2, we have

$$
\begin{aligned}
& \left|\phi_{L_{\varepsilon}(T)}(-u)-\phi_{L(T)}(-u)\right| \\
& \leq|u|\left(b^{2} C_{\theta_{0}} \sigma^{2}(\varepsilon)+\sigma^{2}(\varepsilon)\left(\left|\theta_{0}\right|+C_{\theta_{0}} \sigma^{2}(1)\right)+\sigma^{2}(\varepsilon) C_{\theta_{0}} K_{\theta_{0}}+A\left(\theta_{0}, \varepsilon\right)+\frac{1}{2} \sigma^{2}(\varepsilon)\right) \\
& \quad+C_{\theta_{0}} \sigma^{2}(\varepsilon) K_{\theta_{0}}^{\prime}+B(u, \varepsilon),
\end{aligned}
$$

where $K_{\theta_{0}}=\int_{\varepsilon \leq|z|<1}|z|^{2} e^{z\left|\theta_{0}\right|} \ell(d z), K_{\theta_{0}}^{\prime}=\left|\int_{|z| \geq \varepsilon}\right| e^{i u z}-1-i u z \mathbf{1}_{|z|<1}|| z \mid e^{\left|\theta_{0}\right| z} \ell(d z), A\left(\theta_{0}, \varepsilon\right)=$ $\left|\int_{|z| \leq \varepsilon} z\left(e^{\theta_{0} z}-1\right) \ell(d z)\right|$, and $B(u, \varepsilon)=\left|\int_{|z| \leq \varepsilon}\left(e^{i u z}-1-i u z\right) \ell(d z)\right|$. Moreover, $A\left(\theta_{0}, \varepsilon\right) \leq$ $\left|\theta_{0}\right| e^{\left|\theta_{0}\right|} \sigma^{2}(\varepsilon)$ and $B(u, \varepsilon) \leq u^{2} e^{|u|} \sigma^{2}(\varepsilon)$. Therefore the result follows.

The Greeks are parameters of sensitivity of option prices to the variations of the model descriptive elements, e.g. the Delta and the Gamma are related to the initial condition, the Vega considers the volatility, etc. The next proposition tells us that the Delta of the option price converges.

Proposition 3.5. Under the condition $u \widehat{f}(u) \in L^{1}(\mathbb{R})$, we have

$$
\lim _{\varepsilon \longrightarrow 0} \frac{\partial}{\partial x} \widetilde{\mathbb{E}}_{\theta_{\varepsilon}}\left[f\left(x+L_{\varepsilon}(T)\right]=\frac{\partial}{\partial x} \widetilde{\mathbb{E}}_{\theta_{0}}[f(x+L(T))] .\right.
$$

Proof. We differentiate the integrand in (3.15) and dominate it uniformly in $x$,

$$
\begin{aligned}
\left|\frac{\partial}{\partial x} e^{-i u x} \phi_{L^{\varepsilon}(T)}(u) \widehat{f}(u)\right| & =\left|-i u e^{-i u x} \phi_{L^{\varepsilon}(T)}(u) \widehat{f}(u)\right| \\
& \leq|u \widehat{f}(u)| .
\end{aligned}
$$

Then, by Prop. 2.27 in Folland [10], we can take the derivative operator inside the integral to get

$$
\frac{\partial}{\partial x} \widetilde{\mathbb{E}}_{\theta_{\varepsilon}}\left[f\left(x+L_{\varepsilon}(T)\right]=\frac{1}{2 \pi} \int_{\mathbb{R}}-i u e^{-i u x} \phi_{L_{\varepsilon}(T)}(u) \widehat{f}(u) d u .\right.
$$

Dominating the integrand in the last expression uniformly in $\varepsilon$, the result follows by Prop. 2.24 in Folland [10].

Remark 3.6. Note that we may derive a similar rate of convergence for the delta as we find for the option prices in Prop. 3.4, equation (3.16). 
Remark 3.7. Moreover, we may derive similar convergence results for other Greeks such as the Gamma (the derivative of the delta with respect to the initial condition). In fact we have

$$
\begin{aligned}
\left|\frac{\partial^{2}}{\partial x^{2}} e^{-i u x} \phi_{L^{\varepsilon}(T)}(u) \widehat{f}(u)\right| & =\left|-u^{2} e^{-i u x} \phi_{L^{\varepsilon}(T)}(u) \widehat{f}(u)\right| \\
& \leq\left|u^{2} \widehat{f}(u)\right| .
\end{aligned}
$$

Thus under the condition $u^{2} \widehat{f}(u) \in L^{1}(\mathbb{R})$, we can deduce that the Gamma is robust. Dominating the derivative with respect to the volatility of $\left|e^{-i u x} \phi_{L^{\varepsilon}(T)}(u) \widehat{f}(u)\right|$, we can deduce that the Vega (the derivative of the option price with respect to the volatility) is also robust.

The integrability restriction in the proposition above excludes many interesting examples of functions $f$, like for instance the payoff from a call option. However, we can easily deal with this situation by introducing a damped function $f$ in the following manner. Define for $\alpha>0$ the function

$$
g_{\alpha}(y)=e^{-\alpha y} f(y) .
$$

Assuming that $g_{\alpha} \in L^{1}(\mathbb{R})$ and $\widehat{g}_{\alpha} \in L^{1}(\mathbb{R})$ for some $\alpha>0$, we can apply the above results for $g_{\alpha}$. To translate to $f$, observe that

$$
f(y)=\frac{1}{2 \pi} \int_{\mathbb{R}} e^{(\alpha-\mathrm{i} u) y} \widehat{g}_{\alpha}(u) d u
$$

and

$$
\widehat{g}_{\alpha}(u)=\widehat{f}(u+\mathrm{i} \alpha) .
$$

Hence, Prop. 3.5 holds for any $f$ such that there exists $\alpha>0$ for which we have the following assumption

$$
(\alpha-\mathrm{i} u) \widehat{f}(u+\mathrm{i} \alpha) \in L^{1}(\mathbb{R}) .
$$

Example. We consider an example to illustrate our findings on approximations. Let us assume that $L$ is an NIG-Lévy process, that is, a Lévy process with NIG-distributed increments. Suppose $L(1)$ is NIG distributed with parameters $\mu \in \mathbb{R}, \delta>0, \alpha>0$, $-\alpha \leq \beta \leq \alpha$. We denote by $L(1) \sim \operatorname{NIG}(\mu, \delta, \alpha, \beta)$. The density is (see BarndorffNielsen [3])

$$
p_{\mathrm{NIG}}(x ; \alpha, \beta, \delta, \mu)=\frac{\alpha \delta}{\pi} e^{\delta \sqrt{\alpha^{2}-\beta^{2}}+\beta(x-\mu)} \frac{K_{1}\left(\alpha \sqrt{\delta^{2}+(x-\mu)^{2}}\right)}{\sqrt{\delta^{2}+(x-\mu)^{2}}} .
$$

Here, $K_{1}$ is the modified Bessel function of the second order with parameter 1 , which can be represented by the integral

$$
K_{1}(z)=\frac{\sqrt{\pi} z}{2 \Gamma\left(\frac{3}{2}\right)} \int_{1}^{\infty} e^{-z t}\left(t^{2}-1\right)^{\frac{1}{2}} d t
$$


for $z>0$. The cumulant function is

$$
G(\theta)=\delta\left(\sqrt{\alpha^{2}-\beta^{2}}-\sqrt{\alpha^{2}-\left(\beta^{2}+\theta^{2}\right)}\right)+\mu \theta \text { CMA UNIV. OF OSLO }
$$

which exists for

$$
-\alpha-\beta \leq \theta \leq \alpha-\beta
$$

The Lévy measure $\ell$ is given by

$$
\ell(z)=\frac{\delta \alpha}{\pi} e^{\beta z}|z|^{-1} K_{1}(\alpha|z|) .
$$

In this case $L(t) \sim \operatorname{NIG}(\mu t, \delta t, \alpha, \beta)$ for all $t>0$. If $0<\alpha<\frac{1}{2}$ or $\alpha \geq \frac{1}{2},|\mu|>\delta \sqrt{2 \alpha-1}$, then the Esscher parameter does not exist, however, Hubalek and Sgarra [17] compute analytically the Esscher parameter in the case. $\alpha \geq \frac{1}{2},|\mu| \leq \delta \sqrt{2 \alpha-1}$,

$$
\theta_{0}=-\beta-\frac{1}{2}-\frac{(\mu-r)}{2 \delta} \sqrt{\frac{4 \alpha^{2} \delta^{2}}{(\mu-r)^{2}+\delta^{2}}-1}
$$

Considering the Lévy process $L_{\varepsilon}$, the Esscher parameter $\theta_{\varepsilon}$ exists for $-\alpha-\beta \leq \theta_{\varepsilon} \leq$ $\alpha-\beta-1$. To compute the parameter $\theta_{\varepsilon}$, we consider the fact that

$$
G(\theta)=G_{\varepsilon}(\theta)+\int_{|z|<\varepsilon}\left(e^{\theta z}-1-z \theta\right) \ell(d z)-\frac{1}{2} \theta^{2} \sigma^{2}(\varepsilon),
$$

which leads to

$$
G_{\varepsilon}\left(\theta_{\varepsilon}+1\right)-G_{\varepsilon}\left(\theta_{\varepsilon}\right)=G\left(\theta_{\varepsilon}+1\right)-G\left(\theta_{\varepsilon}\right)+\sigma^{2}(\varepsilon)\left(\theta+\frac{1}{2}\right)+\int_{|z|<\varepsilon}\left(e^{\theta_{\varepsilon} z}\left(1-e^{z}\right)+z\right) \ell(d z) .
$$

The equation (3.6) is therefore equivalent to

$$
G\left(\theta_{\varepsilon}+1\right)-G\left(\theta_{\varepsilon}\right)=r-\sigma^{2}(\varepsilon)\left(\theta_{\varepsilon}+\frac{1}{2}\right)-\int_{|z|<\varepsilon}\left(e^{\theta_{\varepsilon} z}\left(1-e^{z}\right)+z\right) \ell(d z) .
$$

As $\int_{|z|<\varepsilon}\left(e^{\theta_{\varepsilon} z}\left(1-e^{z}\right)+z\right) \ell(d z) \simeq-\theta_{\varepsilon} \sigma^{2}(\varepsilon)$, we find that $\theta_{\varepsilon}$ is approximately the solution of the following equation

$$
G\left(\theta_{\varepsilon}+1\right)-G\left(\theta_{\varepsilon}\right)=r-\frac{1}{2} \sigma^{2}(\varepsilon) .
$$

Using the expression of $G(\theta)$ in (3.19), we get

$$
\theta_{\varepsilon}=-\beta-\frac{1}{2}-\frac{\left(\mu+\frac{1}{2} \sigma^{2}(\varepsilon)-r\right)}{2 \delta} \sqrt{\frac{4 \alpha^{2} \delta^{2}}{\left(\mu+\frac{1}{2} \sigma^{2}(\varepsilon)-r\right)^{2}+\delta^{2}}-1} .
$$

Moreover, we have that the error becomes

$$
\begin{aligned}
\left|\theta_{\varepsilon}-\theta_{0}\right| & =\mid \frac{\mu-r}{2 \delta}\left(\sqrt{\frac{4 \alpha^{2} \delta^{2}}{\left(\mu+\frac{1}{2} \sigma^{2}(\varepsilon)-r\right)^{2}+\delta^{2}}}-1-\sqrt{\frac{4 \alpha^{2} \delta^{2}}{(\mu-r)^{2}+\delta^{2}}-1}\right) \\
& +\frac{\sigma^{2}(\varepsilon)}{\delta} \sqrt{\frac{4 \alpha^{2} \delta^{2}}{\left(\mu+\frac{1}{2} \sigma^{2}(\varepsilon)-r\right)^{2}+\delta^{2}}-1 \mid .}
\end{aligned}
$$




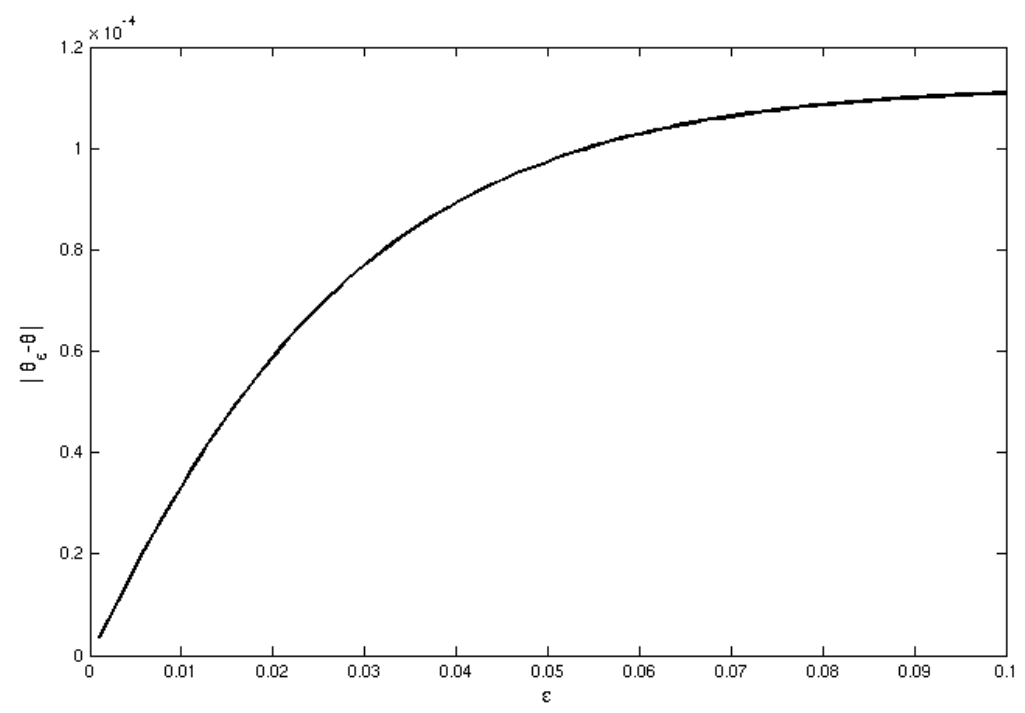

Figure 1. The variation of the error as a function of $\varepsilon$

For a concrete numerical example, let $\alpha=80, \beta=\mu=r=0$, and $\delta=0.03$. The choice of $\alpha$ and $\delta$ here are on the scale relevant for stock prices observed in markets (see for example the estimates in Benth [4] for the NASDAQ and FTSE indices). Figure 1 plots the error $\left|\theta_{\varepsilon}-\theta_{0}\right|$ as a function of $\varepsilon$ for $0<\varepsilon<0.1$. As we can see, it decays fastly to zero, in accordance with our expectations. Even for relatively large $\varepsilon$, the error is rather small. This may be attributed to the fact that an NIG distribution with $\mu=\beta=0$ is symmetric, and very similar to a normal distribution near its center. Notice that in our case the error is analytically given as

$$
\left|\theta_{\varepsilon}-\theta_{0}\right|=\left|\frac{\sigma^{2}(\varepsilon)}{\delta} \sqrt{\frac{4 \alpha^{2} \delta^{2}}{\frac{1}{4} \sigma^{4}(\varepsilon)+\delta^{2}}-1}\right|
$$

Therefore, since $0 \leq \sigma^{2}(\varepsilon) \leq \sigma^{2}(1)$, we have

$$
\frac{\left|\theta_{\varepsilon}-\theta_{0}\right|}{\sigma^{2}(\varepsilon)} \in\left(\frac{1}{\delta} \sqrt{\frac{4 \alpha^{2} \delta^{2}}{\frac{1}{4} \sigma^{4}(1)+\delta^{2}}-1}, \frac{1}{\delta} \sqrt{4 \alpha^{2}-1}\right) .
$$

For our choice of parameters, the interval is very narrow and given by

$$
\frac{\left|\theta_{\varepsilon}-\theta_{0}\right|}{\sigma^{2}(\varepsilon)} \in(0.8333,0.8334),
$$

for $0 \leq \varepsilon \leq 0.1$. Thus, for practical purposes we have an exact error rate rather than an upper bound. 
3.2. The minimal entropy martingale measure. The relative entropy $I_{\mathbb{P}}(\widehat{\mathbb{P}})$ of the measure $\widehat{\mathbb{P}}$ with respect to $\mathbb{P}$ is defined by

$$
I_{\mathbb{P}}(\widehat{\mathbb{P}})= \begin{cases}\mathbb{E}_{\widehat{\mathbb{P}}}\left[\log \frac{d \widehat{\mathbb{P}}}{d \mathbb{P}}\right]=\mathbb{E}_{\mathbb{P}}\left[\frac{d \widehat{\mathbb{P}}}{d \mathbb{P}} \log \frac{d \widehat{\mathbb{P}}}{d \mathbb{P}}\right], & \text { if } \widehat{\mathbb{P}} \ll \mathbb{P} \\ \infty, & \text { otherwise }\end{cases}
$$

The minimal entropy martingale measure is the probability measure that minimizes the value of the function $I_{\mathbb{P}}(\widehat{\mathbb{P}})$ over all $\widehat{\mathbb{P}} \in E M M(\mathcal{P})$. Fujiwara and Miyahara [11] show the existence of the minimal entropy martingale measure for the geometric Lévy process. Moreover, they show that it can be defined by means of the Esscher transform.

Before, we state the theorem by Fujiwara and Miyahara [11], we introduce the following condition on the Lévy process $L$.

$(\mathcal{C})$ : There exists a constant $\theta^{*} \in \mathbb{R}$ that satisfies:

$$
\begin{aligned}
& \left(\mathcal{C}_{1}\right) \int_{|z|>1} e^{z} e^{\theta^{*}\left(e^{z}-1\right)} \ell(d z)<\infty, \\
& \left(\mathcal{C}_{2}\right) a+\left(\frac{1}{2}+\theta^{*}\right) b^{2}+\int_{0<|z| \leq 1}\left\{\left(e^{z}-1\right) e^{\theta^{*}\left(e^{z}-1\right)}-z\right\} \ell(d z)+\int_{|z|>1}\left(e^{z}-1\right) e^{\theta^{*}\left(e^{z}-1\right)} \ell(d z)=r .
\end{aligned}
$$

The next result is due to Fujiwara and Miyahara [11].

Theorem 3.8. Suppose that the condition $(\mathcal{C})$ holds.

(1) We can define a probability measure $\widetilde{\mathbb{P}}$ on $\mathcal{F}_{T}$ by means of the Esscher transform,

$$
\left.\frac{d \widetilde{\mathbb{P}}}{d \mathbb{P}}\right|_{\mathcal{F}_{t}}=\frac{e^{\theta^{*} \widehat{L}(t)}}{\mathbb{E}\left[e^{\theta^{*} \widehat{L}(t)}\right]}=e^{\theta^{*} \widehat{L}(t)-b^{*} t},
$$

where $\widehat{L}(t)$ is the process defined by equation (2.4) and

$$
b^{*}=\frac{\theta^{*}}{2}\left(1+\theta^{*}\right) b^{2}+\theta^{*} a+\int_{\mathbb{R}}\left\{e^{\theta^{*}\left(e^{z}-1\right)}-1-\theta^{*} z \mathbf{1}_{|z| \leq 1}\right\} \ell(d z) .
$$

Thus

$$
\begin{gathered}
\left.\frac{d \widetilde{\mathbb{P}}}{d \mathbb{P}}\right|_{\mathcal{F}_{t}}=\exp \left\{\theta^{*} b W(t)-\frac{1}{2}\left(\theta^{*}\right)^{2} b^{2} t+\theta^{*} \int_{0}^{t} \int_{\mathbb{R}}\left(e^{z}-1\right) \tilde{N}(d s, d z)\right. \\
\left.-t \int_{\mathbb{R}}\left(e^{\theta^{*}\left(e^{z}-1\right)}-1-\theta^{*}\left(e^{z}-1\right)\right) \ell(d z)\right\} .
\end{gathered}
$$

(2) The stochastic process $L$ is still a Lévy process under the probability measure $\widetilde{\mathbb{P}}$ and the characteristic triplet is given by, $\left(b^{2}, \widetilde{\ell}, \widetilde{a}\right)$, where

$$
\tilde{\ell}(d z)=e^{\theta^{*}\left(e^{z}-1\right)} \ell(d z)
$$

and

$$
\widetilde{a}=\theta^{*} b^{2}+a+\int_{|z| \leq 1} z\left(e^{\theta^{*}\left(e^{z}-1\right)}-1\right) \ell(d z) .
$$

Furthermore, the probability measure $\widetilde{\mathbb{P}}$ is in $\operatorname{EMM}(\mathcal{P})$. 
(3) The probability measure $\widetilde{\mathbb{P}}_{\theta^{*}}$ attains the minimal entropy in $\operatorname{ACLM} M(\mathcal{P})$,

$$
\min _{\widehat{\mathbb{P}} \in \operatorname{ALMM(\mathcal {P})}} I_{\mathbb{P}}(\widehat{\mathbb{P}})=I_{\mathbb{P}}\left(\widetilde{\mathbb{P}}_{\theta^{*}}\right) .
$$

Note that $\left(\mathcal{C}_{1}\right)$ is the condition for the moment generating function of the process $\widehat{L}$ to exist. Equation $\left(\mathcal{C}_{2}\right)$ is equivalent to the martingale condition (2.4).

We consider now the Lévy process $L_{\varepsilon}$ which satisfies the following assumption $\left(\mathcal{C}^{\varepsilon}\right)$ similar to $(\mathcal{C})$ before.

$\left(\mathcal{C}^{\varepsilon}\right)$ : There exists $\theta_{\varepsilon}^{*} \in \mathbb{R}$ that satisfies:

$\left(\mathcal{C}_{1}^{\varepsilon}\right) \int_{|z|>1} e^{z} e^{\theta_{\varepsilon}^{*}\left(e^{z}-1\right)} \ell(d z)<\infty$,

$\left(\mathcal{C}_{2}^{\varepsilon}\right)$

$$
\begin{aligned}
a+\left(\frac{1}{2}+\theta_{\varepsilon}^{*}\right)\left(b^{2}+\sigma^{2}(\varepsilon)\right) & +\int_{\varepsilon \leq|z| \leq 1}\left\{\left(e^{z}-1\right) e^{\theta_{\varepsilon}^{*}\left(e^{z}-1\right)}-z\right\} \ell(d z) \\
& +\int_{|z|>1}\left(e^{z}-1\right) e^{\theta_{\varepsilon}^{*}\left(e^{z}-1\right)} \ell(d z)=r .
\end{aligned}
$$

We define a probability measure $\widetilde{\mathbb{P}}_{\theta_{\varepsilon}^{*}}^{\varepsilon}$ by means of the Esscher transform as follows.

$$
\left.\frac{d \widetilde{\mathbb{P}}_{\theta_{\varepsilon}^{*}}^{\varepsilon}}{d \mathbb{P}}\right|_{\mathcal{F}_{t}}=\frac{e^{\theta_{\varepsilon}^{*} \widehat{L}_{\varepsilon}(t)}}{\mathbb{E}\left[e^{\theta_{\varepsilon}^{*} \widehat{L}_{\varepsilon}(t)}\right]}=e^{\theta_{\varepsilon}^{*} \widehat{L}_{\varepsilon}(t)-b_{\varepsilon}^{*} t},
$$

where

$$
\widehat{L}_{\varepsilon}(t)=L_{\varepsilon}(t)+\frac{1}{2}\left(b^{2}+\sigma^{2}(\varepsilon)\right) t+\int_{0}^{t} \int_{|z| \geq \varepsilon}\left(e^{z}-1-z\right) N(d s, d z)
$$

and

$$
b_{\varepsilon}^{*}=\frac{\theta_{\varepsilon}^{*}}{2}\left(1+\theta_{\varepsilon}^{*}\right)\left(b^{2}+\sigma^{2}(\varepsilon)\right)+\theta_{\varepsilon}^{*} a+\int_{|z| \geq \varepsilon}\left\{e^{\theta_{\varepsilon}^{*}\left(e^{z}-1\right)}-1-\theta_{\varepsilon}^{*} z \mathbf{1}_{|z| \leq 1}\right\} \ell(d z) .
$$

Thus

$$
\begin{aligned}
\left.\frac{d \widetilde{\mathbb{P}}_{\theta_{\varepsilon}^{*}}^{\varepsilon}}{d \mathbb{P}}\right|_{\mathcal{F}_{t}}=\exp \left\{\theta_{\varepsilon}^{*}(b W(t)+\sigma(\varepsilon) B(t))-\frac{1}{2}\left(\theta_{\varepsilon}^{*}\right)^{2}\left(b^{2}+\sigma^{2}(\varepsilon)\right) t+\theta_{\varepsilon}^{*} \int_{0}^{t} \int_{|z|>\varepsilon}\left(e^{z}-1\right) \widetilde{N}(d s, d z)\right. \\
\left.\quad-t \int_{|z|>\varepsilon}\left(e^{\theta_{\varepsilon}^{*}\left(e^{z}-1\right)}-1-\theta_{\varepsilon}^{*}\left(e^{z}-1\right)\right) \ell(d z)\right\} .
\end{aligned}
$$

By Theorem 3.8, the probability measure $\widetilde{\mathbb{P}}_{\theta_{\varepsilon}^{*}}^{\varepsilon}$ is the minimal entropy martingale measure for the discounted price process $\widehat{S}_{\varepsilon}$. Moreover, the process $L_{\varepsilon}$ is still a Lévy process under the measure $\widetilde{\mathbb{P}}_{\theta_{\varepsilon}^{*}}^{\varepsilon}$ and the characteristic triplet is given by $\left(b^{2}+\sigma^{2}(\varepsilon), \widetilde{\ell}_{\varepsilon}, \widetilde{a}_{\varepsilon}\right)$, where

$$
\tilde{\ell}_{\varepsilon}(d z)=e^{\theta_{\varepsilon}^{*}\left(e^{z}-1\right)} \ell(d z)
$$


and

$$
\widetilde{a}_{\varepsilon}=\theta_{\varepsilon}^{*}\left(b^{2}+\sigma^{2}(\varepsilon)\right)+a+\int_{\varepsilon \leq|z| \leq 1}^{\mathrm{DEPT} .} z\left(e^{\theta_{\varepsilon}^{*}\left(e^{\varepsilon T H}-1\right)}-1\right) \ell(d z) .
$$

We denote by $\widetilde{E}_{\theta_{\varepsilon}^{*}}[\cdot]$ the expectation with respect to $\widetilde{\mathbb{P}}_{\theta_{\varepsilon}^{* *}}^{\varepsilon}$.

The existence and uniqueness of the solution of equation $\left(\mathcal{C}_{2}\right)$ is proved by Fujiwara and Miyahara [11]. In a similar manner, we can prove the existence and uniqueness of $\theta_{\varepsilon}^{*}$ solution of $\left(\mathcal{C}_{2}^{\varepsilon}\right)$ for $\varepsilon$ fixed in $(0,1)$ and thus we have the following proposition summarizing the result.

Proposition 3.9. Define

$$
F(\theta)=\theta b^{2}+\int_{0<|z| \leq 1}\left(e^{z}-1\right)\left(e^{\theta\left(e^{z}-1\right)}-1\right) \ell(d z)+\int_{|z|>1}\left(e^{z}-1\right) e^{\theta\left(e^{z}-1\right)} \ell(d z),
$$

for $\theta \in(-\infty, \bar{\theta})$, where

$$
\bar{\theta}:=\sup \left\{\theta \in \mathbb{R} ; \int_{|z|>1} e^{z} e^{\theta\left(e^{z}-1\right)} \ell(d z)<\infty\right\} .
$$

Then there exists a unique constant $\theta^{*} \in \mathbb{R}$ satisfying $\left(\mathcal{C}_{2}\right)$ if and only if

$$
r-b_{1} \in\left\{\begin{array}{l}
\left(\lim _{\theta \downarrow-\infty} F(\theta), \lim _{\theta \uparrow \bar{\theta}} F(\theta)\right] \quad \text { in the case when } \bar{\theta}<+\infty \\
\left(\lim _{\theta \downarrow-\infty} F(\theta), \lim _{\theta \uparrow \bar{\theta}} F(\theta)\right) \text { in the case when } \bar{\theta}=+\infty
\end{array}\right.
$$

where $b_{1}=\frac{1}{2} b^{2}+a+\int_{|z| \leq 1}\left(e^{z}-1-z\right) \ell(d z)$.

Define now

$$
F_{\varepsilon}(\theta)=\theta\left(b^{2}+\sigma^{2}(\varepsilon)\right)+\int_{\varepsilon \leq|z| \leq 1}\left(e^{z}-1\right)\left(e^{\theta\left(e^{z}-1\right)}-1\right) \ell(d z)+\int_{|z|>1}\left(e^{z}-1\right) e^{\theta\left(e^{z}-1\right)} \ell(d z),
$$

for $\theta \in(-\infty, \bar{\theta})$. Then for each $\varepsilon \in(0,1)$, there exists a unique constant $\theta_{\varepsilon}^{*} \in \mathbb{R}$ satisfying equation $\left(\mathcal{C}_{2}^{\varepsilon}\right)$ if and only if

$$
r-b_{1}^{\varepsilon} \in\left\{\begin{array}{l}
\left(\lim _{\theta \downarrow-\infty} F_{\varepsilon}(\theta), \lim _{\theta \uparrow \bar{\theta}} F_{\varepsilon}(\theta)\right] \quad \text { in the case when } \bar{\theta}<+\infty \\
\left(\lim _{\theta \downarrow-\infty} F_{\varepsilon}(\theta), \lim _{\theta \uparrow \bar{\theta}} F_{\varepsilon}(\theta)\right) \text { in the case when } \bar{\theta}=+\infty,
\end{array}\right.
$$

where $b_{1}^{\varepsilon}=\frac{1}{2}\left(b^{2}+\sigma^{2}(\varepsilon)\right)+a+\int_{\varepsilon \leq|z| \leq 1}\left(e^{z}-1-z\right) \ell(d z)$.

By the same argument as in Lemma 3.1 and under the conditions (3.23) and (3.24), we can prove that $\theta_{\varepsilon}^{*}$ is bounded uniformly in $\varepsilon$ and that

$$
\left|\theta_{\varepsilon}^{*}-\theta^{*}\right| \leq C_{\theta^{*}} \sigma^{2}(\varepsilon) \text { and }\left|\theta_{\varepsilon}^{*}\right| \leq\left|\theta^{*}\right|+C_{\theta^{*}} \sigma^{2}(1) .
$$

Thus, we have the following result concerning stability of option pricing.

Proposition 3.10. For $f \in L^{1}(\mathbb{R})$, we have

$$
\lim _{\varepsilon \rightarrow 0} \widetilde{\mathbb{E}}_{\theta_{\varepsilon}^{*}}\left[f\left(x+L_{\varepsilon}(T)\right]=\widetilde{\mathbb{E}}_{\theta^{*}}[f(x+L(T)] .\right.
$$


Proof. Recall from (3.15) that

$$
\widetilde{\mathbb{E}}_{\theta_{\varepsilon}^{*}}\left[f\left(x+L_{\varepsilon}(T)\right]=\frac{1}{2 \pi} \int_{\mathbb{R}} e^{-i u x} \phi_{L_{\varepsilon}(T)}(-u) \widehat{f}(u) d u .\right.
$$

From the characteristic triplet of the process $L_{\varepsilon}$ under the measure $\widetilde{\mathbb{P}}_{\theta_{\varepsilon}^{*}}^{\varepsilon}$, we can write the characteristic function $\phi_{L_{\varepsilon}(T)}(u)$ as follows:

$$
\phi_{L_{\varepsilon}(T)}(u)=\exp \left\{i \widetilde{a}_{\varepsilon} u-\frac{1}{2}\left(b^{2}+\sigma^{2}(\varepsilon)\right) u^{2}+\int_{|z| \geq \varepsilon}\left\{e^{i u z}-1-i u z \mathbf{1}_{|z|<1}\right\} \widetilde{\ell}_{\varepsilon}(d z)\right\} .
$$

As $\theta_{\varepsilon}^{*}$ is bounded and converges to $\theta^{*}$ then $\phi_{L_{\varepsilon}(T)}(u)$ converges to $\phi_{L(T)}(u)$, for all $u \in \mathbb{R}$, where $\phi_{L(T)}(u)$ is the characteristic function of $L$ under the measure $\widetilde{\mathbb{P}}_{\theta^{*}}$. Taking the limit inside the integral in equation (3.25), the result follows.

Using the same arguments as in Prop 3.5, we can also show that the delta of the option price converges and we have the following proposition.

Proposition 3.11. Under the condition $u \widehat{f}(u) \in L^{1}(\mathbb{R})$, we have

$$
\lim _{\varepsilon \longrightarrow 0} \frac{\partial}{\partial x} \widetilde{\mathbb{E}}_{\theta_{\varepsilon}^{*}}\left[f\left(x+L_{\varepsilon}(T)\right]=\frac{\partial}{\partial x} \widetilde{\mathbb{E}}_{\theta^{*}}[f(x+L(T))] .\right.
$$

Note that, by using the Fourier transform of the function $f$ and the mean value theorem, we may derive convergence rates as well for these two results, analogous to the Esscher transform case.

3.3. The minimal martingale measure. In this section, we assume that the Lévy measure of the process $L$ satisfies the following integrability conditions

$$
\int_{z>1} e^{2 z} \ell(d z)<\infty
$$

Recall the dynamics of $\widehat{S}$ in (2.5). Since it is a semimartingale, we can decompose it into a local martingale $M$ and a finite variation process $A$, with $A(0)=0$, where $M$ and $A$ have the following expressions

$$
\begin{gathered}
M(t)=\widehat{S}(0)+\int_{0}^{t} b \widehat{S}(s-) d W(s)+\int_{0}^{t} \int_{\mathbb{R}_{0}} \widehat{S}(s-)\left(e^{z}-1\right) \widetilde{N}(d s, d z), \\
A(t)=\int_{0}^{t}\left(a_{1}-r\right) \widehat{S}(s) d s .
\end{gathered}
$$

We denote by $\langle X\rangle$ the predictable compensator of the process $X$. Then, we have

$$
\langle M\rangle(t)=\int_{0}^{t} b^{2} \widehat{S}^{2}(s) d s+\int_{0}^{t} \int_{\mathbb{R}_{0}} \widehat{S}^{2}(s)\left(e^{z}-1\right)^{2} \ell(d z) d s
$$

and we can represent the process $A$ as follows

$$
A(t)=\int_{0}^{t} \frac{a_{1}-r}{b^{2} \widehat{S}(s)+\widehat{S}(s) \int_{\mathbb{R}_{0}}\left(e^{z}-1\right)^{2} \ell(d z)} d\langle M\rangle(s) .
$$


Let $\alpha$ be the integrand in equation (3.29), that is, the predictable process given by

$$
\alpha(t):=\frac{a_{1}-r^{\text {DEPT. OF MATH./CMA UNIV. }}}{b^{2} \widehat{S}(t)+\widehat{S}(t) \int_{\mathbb{R}_{0}}\left(e^{z}-1\right)^{2} \ell(d z)}, \quad 0 \leq t \leq T .
$$

We define a process $K$ by means of $\alpha$ as follows

$$
K(t)=\int_{0}^{t} \alpha^{2}(s) d\langle M\rangle(s)=\frac{\left(a_{1}-r\right)^{2}}{b^{2}+\int_{\mathbb{R}_{0}}\left(e^{z}-1\right)^{2} \ell(d z)} t .
$$

The process $K$ is called the mean-variance trade-off process. The processes defined above will be used later in connection with our analysis of the minimal martingale measure. Under the condition (3.26), the local martingale part $M$ defined in (3.27) is a square integrable $\mathbb{P}$-martingale and the stock price process $\widehat{S}$ is a special semimartingale (see Schweizer [21]). Moreover, for any $\widetilde{\mathbb{P}} \in A C L M M(\mathbb{P})$, the process $\widehat{S}$ is not only a local martingale but a martingale under $\widetilde{\mathbb{P}}$.

The notion of minimal martingale measure was introduced in Föllmer and Schweizer [12]. A martingale measure $\widetilde{\mathbb{P}}$ is called minimal if any square-integrable $\mathbb{P}$-martingale which is orthogonal to the martingale part of $\widehat{S}$ under $\mathbb{P}$ remains a martingale under $\widetilde{\mathbb{P}}$. Föllmer and Schweizer [12] show the existence and uniqueness of this measure in the case of special semimartingales (see Schweizer [21]). The condition (3.26) ensures the existence and uniqueness of the minimal martingale measure in our model, and we have the next result given in Prop 4.1 in Arai [1].

Theorem 3.12. The following holds;

(1) We define a probability measure $\widetilde{\mathbb{P}}$ on $\mathcal{F}_{T}$ by

$$
\left.\frac{d \widetilde{\mathbb{P}}}{d \mathbb{P}}\right|_{\mathcal{F}_{t}}=\exp \left\{-\int_{0}^{t} \alpha(s) d M(s)-\frac{1}{2} K(t)\right\}
$$

where the processes $\alpha, M$ and $K$ are defined by equations (3.30), (3.27) and (3.31) respectively. Denote by $\gamma=\frac{\alpha(s)}{\widehat{S}(s)}=\frac{a_{1}-r}{b^{2}+\int_{\mathbb{R}}\left(e^{z}-1\right)^{2} \ell(d z)}$. Then

$$
\left.\frac{d \widetilde{\mathbb{P}}}{d \mathbb{P}}\right|_{\mathcal{F}_{t}}=\exp \left\{-\int_{0}^{t} \gamma b d W(s)-\int_{0}^{t} \int_{\mathbb{R}_{0}} \gamma\left(e^{z}-1\right) \tilde{N}(d s, d z)-\frac{1}{2} K(t)\right\} .
$$

(2) The stochastic process $L$ is still a Lévy process under the probability measure $\widetilde{\mathbb{P}}$ and the characteristic triplet is given by $\left(b^{2}, \widetilde{\ell}, \widetilde{a}\right)$, where

$$
\tilde{\ell}(d z)=\left\{\left(e^{z}-1\right) \gamma+1\right\} \ell(d z)
$$

and

$$
\widetilde{a}=a+b^{2} \gamma+\int_{|z| \leq 1} \gamma z\left(e^{z}-1\right) \ell(d z) .
$$

(3) The density process $\frac{d \widetilde{\mathbb{P}}}{d \mathbb{P}}$ is a square integrable martingale.

(4) The measure $\widetilde{\mathbb{P}}$ is a minimal martingale measure. 
Next we consider the approximating price process $S_{\varepsilon}$. Following the theorem above and use the corresponding definitions of the involved parameters and functions. Hence we define $\gamma_{\varepsilon}$ by

$$
\gamma_{\varepsilon}=\frac{c_{\varepsilon}}{b^{2}+\sigma^{2}(\varepsilon)+\int_{|z| \geq \varepsilon}\left(e^{z}-1\right)^{2} \ell(d z)}
$$

where

$$
c_{\varepsilon}=a+\frac{1}{2}\left(b^{2}+\sigma^{2}(\varepsilon)\right)+\int_{|z| \geq \varepsilon}\left(e^{z}-1-z \mathbf{1}_{|z| \leq 1}\right) \ell(d z)-r .
$$

Let $\widetilde{\mathbb{P}}^{\varepsilon}$ be a measure which is absolutely continuous with respect to $\mathbb{P}$ such that

$$
\left.\frac{d \widetilde{\mathbb{P}}^{\varepsilon}}{d \mathbb{P}}\right|_{\mathcal{F}_{t}}=\exp \left\{-\int_{0}^{t} \gamma_{\varepsilon} \sqrt{b^{2}+\sigma^{2}(\varepsilon)} d \widetilde{B}_{\varepsilon}(s)-\int_{0}^{t} \int_{|z|>\varepsilon} \gamma_{\varepsilon}\left(e^{z}-1\right) \tilde{N}(d s, d z)-\frac{1}{2} K_{\varepsilon}(t)\right\}
$$

where

and

$$
\widetilde{B}_{\varepsilon}(t)=\frac{b}{\sqrt{b^{2}+\sigma^{2}(\varepsilon)}} W(t)+\frac{\sigma(\varepsilon)}{\sqrt{b^{2}+\sigma^{2}(\varepsilon)}} B(t)
$$

$$
K_{\varepsilon}(t)=\frac{c_{\varepsilon}^{2}}{b^{2}+\sigma^{2}(\varepsilon)+\int_{|z|>\varepsilon}\left(e^{z}-1\right)^{2} \ell(d z)} .
$$

By Theorem 3.12 and under the condition (3.26), the measure $\widetilde{\mathbb{P}^{\varepsilon}}$ is a minimal martingale measure for the discounted price process $\widehat{S}_{\varepsilon}$. Moreover, the stochastic process $L_{\varepsilon}$ is still a Lévy process under $\widetilde{\mathbb{P}}^{\varepsilon}$ and the characteristic triplet is given by $\left(b^{2}+\sigma^{2}(\varepsilon), \widetilde{\ell}_{\varepsilon}, \widetilde{a}_{\varepsilon}\right)$, where

$$
\tilde{\ell}_{\varepsilon}(d z)=\left\{\left(e^{z}-1\right) \gamma_{\varepsilon}+1\right\} \mathbf{1}_{|z| \geq \varepsilon} \ell(d z),
$$

and

$$
\widetilde{a}_{\varepsilon}=a+\left(b^{2}+\sigma^{2}(\varepsilon)\right) \gamma_{\varepsilon}+\int_{\varepsilon \leq|z| \leq 1} \gamma_{\varepsilon} z\left(e^{z}-1\right) \ell(d z) .
$$

Denote by $\widetilde{\mathbb{E}}_{\varepsilon}[\cdot]$ the expectation with respect to the measure $\widetilde{\mathbb{P}} \varepsilon$.

We have the following convergence result for option prices.

Proposition 3.13. For $f \in L^{1}(\mathbb{R})$, we have

$$
\lim _{\varepsilon \rightarrow 0} \widetilde{\mathbb{E}}_{\varepsilon}\left[f\left(x+L_{\varepsilon}(T)\right]=\widetilde{\mathbb{E}}[f(x+L(T)] .\right.
$$

Proof. Recall from (3.15) that

$$
\widetilde{\mathbb{E}}_{\varepsilon}\left[f\left(x+L_{\varepsilon}(T)\right]=\frac{1}{2 \pi} \int_{\mathbb{R}} e^{-i u x} \phi_{L_{\varepsilon}(T)}(u) \widehat{f}(u) d u\right.
$$

From the characteristics of the process $L_{\varepsilon}$ under the measure $\widetilde{\mathbb{P}} \varepsilon$, we can prove that the characteristic function $\phi_{L_{\varepsilon}(T)}(u)$ converges to $\phi_{L(T)}(u)$, for all $u \in \mathbb{R}$, where $\phi_{L(T)}(u)$ is the characteristic function of $L$ under the measure $\widetilde{\mathbb{P}}$. Taking the limit inside the integral in equation (3.37), we obtain the result. 
Remark 3.14. Let us define $D(\widehat{\mathbb{P}}, \mathbb{P})$ as

$$
D(\widehat{\mathbb{P}}, \mathbb{P})=\sqrt{\operatorname{Var}\left(\frac{d \widehat{\mathbb{P}}}{d \mathbb{P}}\right)}, \quad \widehat{\mathbb{P}} \in A L L M(\mathcal{P}) .
$$

A probability measure $\widetilde{\mathbb{P}}$ is the mean-variance martingale measure if it minimizes $D(\widehat{\mathbb{P}}, \mathbb{P})$ over all $\widehat{\mathbb{P}} \in A L L M(\mathcal{P})$. In Theorem 8 in Schweizer [21], it is shown that the meanvariance martingale measure coincides with the minimal martingale measure if the following conditions hold:

- The price process $\widehat{S}$ is decomposed into a martingale process and a finite variation process.

- The finite variation process $A$ is absolutely continuous with respect to $\langle M\rangle$.

- The mean-variance trade-off process $K$ is deterministic.

In our model, these conditions are satisfied. Our convergence results for the minimal martingale measure transfer to the mean-variance measure as well.

\section{Conclusions}

Our results show that option prices are stable with respect to perturbation in the underlying dynamics when we substitute small jumps with an appropriately scaled Brownian motion. In practical terms, we may interpret this as having two competing models, one where we suppose that small variations in the asset dynamics come from a jump process of infinite activity, and another where we model this by a Brownian motion. It is very hard, if possible, to decide which model is better from a statistical point of view. However, the result above shows that the effect on option prices is very small.

From a different perspective, if we want to perform a numerical evaluation of the option price, we may apply the above result in order to quantify the error if we approximate small jumps by a Brownian motion. The error is explicit in terms of $\sigma(\varepsilon)$, that is the volatility of the jumps smaller than $\varepsilon$. Moreover, from the simulation point of view, it is well known that in practice it is difficult to simulate from a Lévy process $L$ directly. The approximating process consists of a Brownian motion and a compound Poisson process which are both processes easy to simulate. In summary our study aims at bridging the gap between theory and practice providing the grounds for a proper use of numerical methods.

Based on the accomplishments we presented, one can discretize the approximating Lévy process for instance, by an Euler scheme and combine the approximation and the discretization to derive an estimation of the model risk. In fact, in practice one deals with actions discrete in time while theory develops models continuous in time. In this issue one can explore the discretization error for Lévy models and also study the combined effect (approximation and discretization).

\section{REFERENCES}

[1] Arai, T. (2001). On the equivalent martingale measures for Poisson jump type model. J. Appl. Prob., 38, pp. 482-493. 
[2] Asmussen, S., and Rosinski, J. (2001). Approximations of small jump Lévy processes with a view towards simulation. J. Appl. Prob., 38, pp. 482-493.

[3] Barndorff-Nielsen, O.E. (1998). Processes of normal inverse Gaussian type. Finance Stoch., 2(1), pp. 41-68.

[4] Benth, F. E. (2004). Option Theory with Stochastic Analysis, Springer-Verlag, Heidelberg.

[5] Benth, F. E., Di Nunno, G., and Khedher, A. (2010). Lévy-models robustness and sensitivity. In Volume XXV of the series $Q P-P Q$, Quantum Probability and White Noise Analysis, H. Ouerdiane and A. Barhoumi (eds.), World Scientific, pp. 153-184.

[6] Benth, F. E., Di Nunno, G., and Khedher, A. (2011). Robustness of option prices and their deltas in markets modelled by jump-diffusions. Comm. Stoch. Analysis, 5(2), pp. 285307.

[7] Bühlmann, H., Delbaen, F., Embrechts, P., and Shirayev, A.N. (1996). No-arbitrage, change of measure and conditional Esscher transforms. CWI Quarterly 9., 4, pp. 291-317.

[8] Carr, P., and Madan D. B. (1998). Option valuation using fast Fourier transform. J. Comp. Finance, 2, pp. 61-73.

[9] Cont, R., and Tankov, P. (2004). Financial Modelling with Jump Processes. Chapman Hall.

[10] Folland, G. B. (1984). Real Analysis - Modern Techniques and their Applications. John Wiley \& Sons.

[11] Fujiwara, T., and Miyahara, Y. (2003). The minimal entropy martingale measures for geometric Lévy processes, Finance Stoch., 7, pp. 509-531.

[12] Föllmer, H., and Schweizer, M. (1991). Hedging of contingent claims under incomplete information. In Applied Stochastic Analysis., M.H.A. Davis and R.J. Elliot (eds.), Stochastic Monograhps, Gordon and Breach London-New York, 5, pp. 389-414.

[13] Föllmer, H., and Sondermann, D. (1986). Hedging of non-redundant contingent claims. Contributions to Mathematical Economics. W. Hildebrand and A. Mas-Collel (eds.), Elsevier Science Publishers B.V. North Holland, pp. 205-223.

[14] Gerber, H.U., and Shiu, E.S.W. (1994). Option pricing by Esscher transforms. Trans. Soc. Actuaries, 46, pp. 99-191.

[15] Gerber, H.U., and Shiu, E.S.W. (1996). Actuarial bridges to dynamic hedging and option pricing. Insurance: Math. Economics, 18, pp. 183-218.

[16] Goll, T., and Rüschendorf, L. (2001). Minimax and minimal distance martingale measures and their relationship to portfolio optimization. Finance Stoch., 5 pp. 557-581.

[17] Hubalek, F., Sgarra, C (2006). Esscher transforms and the minimal entropy martingale measure for exponential Lévy models. Quantitative Finance, 6, pp. 125-145.

[18] Karatzas, I., and Shreve, S. E. (1991). Brownian Motion and Stochastic Calculus. Springer.

[19] Rydberg, T. H. (1997). The normal inverse Gaussian Lévy process: simulation and approximation. Comm. Stat. Stoch. Models, 13, pp. 887-910.

[20] Sato, K.-I. (1999). Lévy Processes and Infinitely Divisible Distributions. Cambridge University Press.

[21] Schweizer. M. (1994). On the minimal martingale measure and the Follmer-Schweizer decomposition, Stoch. Analysis Appl., 13, pp. 573-599..

[22] Tankov, P. (2011). Pricing and hedging in exponential Lévy models: review of recent results. In Paris-Princeton Lecture Notes in Mathematical Finance, R. Carmona et al. (eds.), Springer Verlag, pp. 319-370. 
Fred Espen Benth: Center of Mathematics for Applications, University of Oslo, PO Box 1053 Blindern, N-0316 Oslo, Norway DePt. OF MATH./CMA UNIV. OF OSLO

Giulia Di Nunno: Center of Mathematics for Applications, University of Oslo, PO Box 1053 Blindern, N-0316 Oslo, Norway, And, Norwegian School of Economics and Business Administration, Helleveien 30, N-5045 Bergen, Norway.

Asma Khedher: Center of Mathematics for Applications, University of Oslo, PO Box 1053 Blindern, N-0316 Oslo, NorWAy

E-mail address: [fredb,giulian] @math.uio.no, asma.khedher@cma.uio.no

URL: http://folk.uio.no/[fredb,giulian]/ 